\title{
Emerging role of long non-coding RNAs in cisplatin resistance
}

This article was published in the following Dove Press journal:

OncoTargets and Therapy

\author{
Yang $\mathrm{Hu}^{1,2}$ \\ Qiong-Ni Zhu',2 \\ Jun-Li Deng ${ }^{1,2}$ \\ Zhi-Xing $\mathrm{Li}^{1,2}$ \\ Guo Wang ${ }^{1,2}$ \\ Yuan-Shan Zhu ${ }^{3}$ \\ 'Department of Clinical \\ Pharmacology, Xiangya Hospital, \\ Central South University, Changsha, \\ Hunan, People's Republic of China; \\ 'Institute of Clinical Pharmacology, \\ Central South University, Hunan Key \\ Laboratory of Pharmacogenetics, \\ Changsha, Hunan, People's Republic of \\ China; ${ }^{3}$ Department of Medicine, Weill \\ Cornell Medicine, New York, NY, USA
}

Correspondence: Guo Wang

Department of Clinical Pharmacology,

Xiangya Hospital, Institute of Clinical

Pharmacology, Central South University,

II 0 Xiangya Road, Changsha, Hunan,

410008, People's Republic of China

$\mathrm{Tel}+8673184805380$

Email 207082@csu.edu.cn

Yuan-Shan Zhu

Department of Medicine, Weill Cornell

Medicine, 1300 York Avenue, Box 149,

New York, NY 10065, USA

Tel + I 2127464745

Email yuz2002@med.cornell.edu

\begin{abstract}
Cisplatin (CDDP) is one of the most commonly used chemotherapy drugs for the treatment of various cancers. Although platinum-based therapies are highly efficacious against rapidly proliferating malignant tumors, the development of CDDP resistance results in significant relapse as well as decreased overall survival rates, which is a significant obstacle in CDDP-based cancer therapy. Long non-coding RNAs (lncRNAs) are involved in cancer development and progression by the regulation of processes related to chromatin remodeling, transcription, and posttranscriptional processing. Emerging evidence has recently highlighted the roles of lncRNAs in the development of CDDP resistance. In this review, we discuss the roles and mechanisms of lncRNAs in CDDP chemoresistance, including changes in cellular uptake or efflux of a drug, intracellular detoxification, DNA repair, apoptosis, autophagy, cell stemness, and the related signaling pathways, aiming to provide potential lncRNA-targeted strategies for overcoming drug resistance in cancer therapy.

Keywords: cisplatin, lncRNAs, chemoresistance, cancer
\end{abstract}

\section{Introduction}

Cancer significantly affects the quality of life and is a leading cause of death worldwide. It has been reported that about 14.1 million new cancer cases and 8.2 million deaths occurred in 2012 worldwide. ${ }^{1}$ In 2018, 1,735,350 new cancer cases and 609,640 cancer deaths are projected to occur in the USA. ${ }^{2}$ Increasing national investment in cancer research contributes to accelerating progress in the prevention and treatment of cancer. Currently, the gold standard for antitumor therapeutic strategies is a combination of chemotherapy and surgery. However, chemotherapeutic anticancer agents are the standard treatment regimen for patients in whom surgery is not a viable option. ${ }^{3}$ Cisplatin is one of the most widely used and successful cytotoxic drugs for the treatment of a broad variety of tumors such as ovarian, testicular, bladder, lung, esophageal, and nasopharyngeal carcinoma (NPC). Since the discovery of the antitumor activity of cisplatin, novel platinum-based agents (carboplatin and oxaliplatin) have been developed with reduced side effects and increased efficacy. ${ }^{4}$ However, as a prototype of platinum-based agent, cisplatin remains widely used as a chemotherapeutic agent. When cisplatin is used in platinum-based chemotherapy, nearly $85 \%$ of patients with metastatic testicular cancer can be cured ${ }^{5}$ and the 5-year survival rate in patients with completely resected non-small-cell lung cancer (NSCLC) tumors is improved. ${ }^{6}$

Nevertheless, there exist many patients intrinsically resistant to cisplatin-based therapies, especially with colorectal, lung, and prostate cancers. What is more, originally sensitive tumors eventually develop chemoresistance, which is frequently observed in ovarian cancer. ${ }^{7}$ Chemoresistance allows the cancer cells to become increasingly antagonistic and improves the ability of cancer invasion and migration, leading to tumor relapse 
and poor prognosis. ${ }^{8,9}$ Emerging studies have revealed that dysregulated expression of long non-coding RNAs (lncRNAs) plays an essential role in cisplatin resistance. ${ }^{10}$ The lncRNAs, which are $>200$ nucleotides (nt) in length and which lack a significant open reading frame, may play major roles in a wide variety of biological pathways and cellular processes at the epigenetic, transcriptional, and posttranscriptional levels. ${ }^{10,11}$ Here, we briefly review the functions and mechanisms of lncRNAs in the regulation of drug resistance in cancer cells, mainly focusing on cisplatin chemoresistance.

\section{Cisplatin}

As an alkylating agent, cisplatin was first described by Michele Peyrone in 1845, and its antitumor activity was discovered in the 1970 s. ${ }^{12,13}$ Since its approval by the US Food and Drug Administration for the treatment of testicular and ovarian cancer in $1987,{ }^{12,14}$ cisplatin has gradually become a first-line chemotherapeutic agent. The platinum atom of cisplatin interacts with nucleophilic $\mathrm{N}^{7}$-sites of purine in DNA to form inter- and intra-strand DNA crosslinks, ${ }^{8,14}$ which results in DNA damage, cell cycle arrest, and activation of multiple signal transduction pathways, leading to cell apoptosis. ${ }^{8,15}$ Moreover, cisplatininduced production of reactive oxygen species and activation of inflammatory pathways may also contribute to the induction of apoptosis. ${ }^{16}$ The introduction of cisplatin for the treatment of testicular cancer has improved its cure rate from $10 \%$ to $85 \%$. ${ }^{17}$ Unfortunately, the development of cisplatin resistance limits its efficacy in cancer treatment. Studies over the years have revealed multiple potential mechanisms related to cisplatin resistance (Figure 1). Cisplatin resistance may occur through reduced intracellular platinum accumulation due to decreased drug uptake or increased drug export in cancer cells. Downregulation of copper transporter 1 (CTR1) has been associated with resistance to cisplatin by reducing cisplatin uptake. ${ }^{18}$ On the other hand, the efflux of cisplatin is mediated by transporting P-type adenosine triphosphatases (ATP) 7A and ATP7B, or multidrug-resistance-associated proteins (MRPs) in the cell membrane, and an upregulation of these efflux transporters is one of the major mechanisms of cisplatin resistance. ${ }^{19} \mathrm{Cispla-}$ tin scavenging by intracellular detoxification is another major mechanism of cisplatin resistance, in which glutathione (GSH) plays a key role in the overexpression of enzymes involved in GSH synthesis and GSH conjugation has been reported to be associated with cisplatin resistance. ${ }^{20}$ In addition, activation of the DNA damage systems, such as the nucleotide excision repair system, can attenuate the apoptotic process, leading

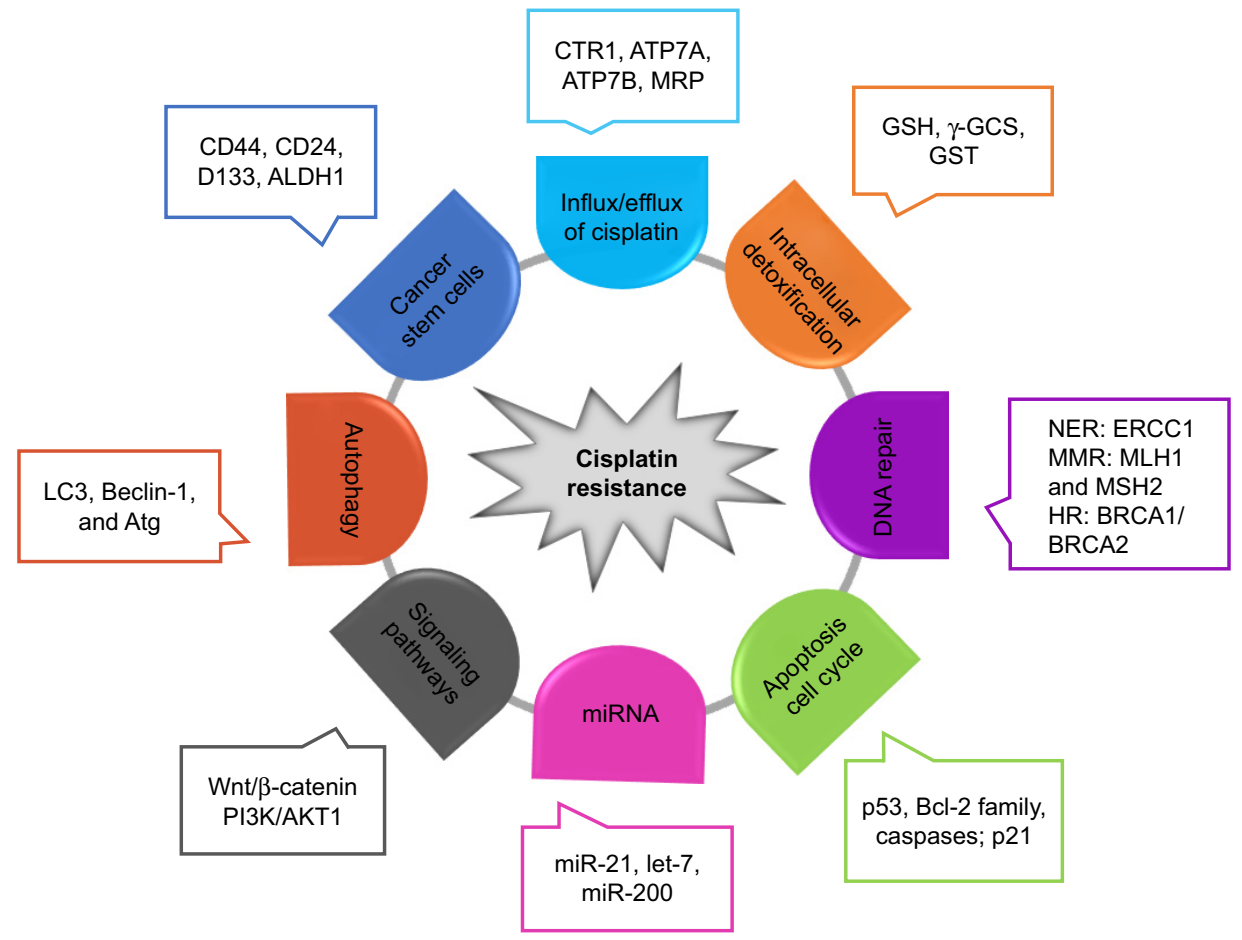

Figure I Molecular mechanisms of cisplatin resistance.

Notes: Multiple cellular alterations in cancer cells, including cell cycle, apoptosis, autophagy, stemness, intracellular detoxification, and drug influx/efflux, contribute to cisplatin chemoresistance through genetic and/or epigenetic regulation of multiple signaling pathways. Some major genetic and epigenetic factors are illustrated in the figure (see text for detailed discussion).

Abbreviations: ALDHI, aldehyde dehydrogenase I family member AI; ATG7, autophagy associated gene; BRCA2, breast cancer susceptibility proteins 2; CTRI, copper transporter I; ERCCI, excision repair cross-complementing rodent repair deficiency, complementation group I; GSH, glutathione; GST, glutathione S-transferase; HR, homologous recombination; MMR, mismatch repair; MRP, multidrug-resistant-associated protein; NER, nucleotide excision repair; $\gamma$-GCS, $\gamma$-glutamylcysteine synthetase. 
to cisplatin resistance. Increased expression of nucleotide excision repair proteins, including XPF-ERCC1 complex, is associated with reduced efficacy of platinum-based therapy. ${ }^{21}$ Since the mismatch repair (MMR) system can detect cisplatin-induced DNA lesion and activate the apoptotic signal, downregulation or a mutation of MMR-related genes such as $\mathrm{MLH1}$ and $\mathrm{MSH} 2$ has been reported to contribute to cisplatin resistance. ${ }^{22}$ Homologous recombination is another mechanism to repair cisplatin-induced DNA damage, and hence, a deficiency of breast cancer susceptibility proteins 1 and 2 (BRCA1/2), two critical components in the homologous recombination system, promotes cell sensitivity to cisplatin in cancer cells. ${ }^{23}$ The expression of tumor suppressor protein $\mathrm{p} 53$ and p53-related nuclear transcription factors in cancer cells has been shown to mediate the cytotoxic effect of cisplatin. ${ }^{24,25}$ As the cytotoxic effect of cisplatin is associated with apoptotic signaling pathways, the expression levels of $\mathrm{Bcl}-2$ proteins, caspases, and mitochondrial intermembrane proteins are crucial factors in influencing cell sensitivity to cisplatin. ${ }^{26-28}$ Furthermore, accumulating evidence suggests that the alteration in cell autophagy and PI3K/AKT1 signaling pathway can modulate cell sensitivity to cisplatin through compensating for cisplatin-induced lethal signals..$^{29,30}$

\section{IncRNA}

With the rapid development of sequencing technologies, it has been determined that $<2 \%$ of the human genome encodes proteins, while the remaining transcriptional products are ncRNAs, which are considered as non-functional and transcriptional noise. ${ }^{31}$ The ncRNAs can be classified into two major groups based on their sizes: small ncRNAs for those with a length $<200 \mathrm{nt}$ and lncRNAs for those with a length $>200 \mathrm{nt}$, which includes intronic lncRNAs, intergenic lncRNAs, bidirectional lncRNAs, enhancer lncRNAs, and sense or antisense lncRNAs. ${ }^{32}$ The lncRNAs can modulate gene expression at epigenetic, transcriptional, and posttranscriptional levels. ${ }^{10,33}$ In recent years, various studies have suggested that lncRNAs are involved in embryonic development and in the etiology of many human diseases, especially cancer. ${ }^{34}$ Using advanced sequencing technology, numerous lncRNAs have been found to be dysregulated or aberrantly expressed in multiple types of cancers. The lncRNAs have been reported to act as critical factors in cancer development and progression by regulating cell proliferation, cell death, metastasis, and angiogenesis. ${ }^{35}$ As lncRNAs play an important role in tumor cell survival and death, it is conceivable that lncRNAs may also alter cell sensitivity to chemotherapy, which is aimed at eradicating tumor cells by inhibiting cell growth and promoting cell apoptosis. It has been reported that lncRNA H19 contributes to doxorubicin resistance through regulating MDR1 expression. ${ }^{36} \mathrm{Du}$ et al have reported that lncRNA-XIST promoted temozolomide resistance in glioma cells through DNA MMR pathway. ${ }^{37}$ Moreover, lncRNA UCA1 has been shown to promote 5-fluorouracil resistance in colorectal cancer cells by inhibiting miR-204-5p. ${ }^{38}$ In sum, growing evidence has indicated that dysregulated expression of lncRNAs in cancer cells plays an important role in the development of chemoresistance through altering the mechanisms of drug export, drug metabolism, DNA repair, cell proliferation, apoptosis, and autophagy.,11

\section{IncRNA and cisplatin resistance}

As stated above, numerous studies over the years have demonstrated that lncRNAs play a significant role in chemoresistance. ${ }^{11}$ Aiming to understand the roles and mechanisms of lncRNAs in cisplatin resistance, we searched PubMed for all articles associated with "IncRNA and cisplatin resistance" and found that 22 lncRNAs have been reported to play an important role in cisplatin resistance through various mechanisms in multiple cancers (Table 1; Figure 2).

\section{Influx/efflux of cisplatin}

Previous studies have indicated that reduced drug uptake or increased drug efflux in cancer cells, which results in a reduced intracellular platinum accumulation, is an important biochemical and cytological mechanism of cisplatin resistance. ${ }^{8}$ ATP-binding cassette transporters, including P-glycoprotein and MRPs, can increase the drug efflux. Hang et $\mathrm{al}^{39}$ have reported that Notch 1 could promote cisplatin resistance in gastric cancer (GC) through upregulation of lncRNA AK022798 expression. When lncRNA AK022798 was knocked down, the expression of MRP1 and P-glycoprotein MDR1, two membrane drug efflux-porters, was significantly decreased, while cell apoptosis, caspase-3, and caspase- 8 activities were significantly increased in SGC7901 and BGC823 cisplatin-resistant cancer cells. These results indicated that lncRNA AK022798 is a crucial mediator in Notch 1-induced cisplatin resistance in cancer cells. ${ }^{39}$ In GC tissues and cells, high expression of lncRNA PVT1 was significantly associated with the development of cisplatin resistance. ${ }^{40}$ PVT1 silencing could reverse the cisplatin resistance in cisplatin-resistant cell lines, while upregulation of PVT1 decreased the sensitivity of parental GC cells to cisplatin, which was mediated through upregulation of MDR1, MRP, mTOR, and HIF-1a expression. ${ }^{40}$ The lncRNA ANRIL has also been reported to be highly expressed in 
Table I Predictive IncRNAs involved in response to cisplatin

\begin{tabular}{|c|c|c|c|c|c|}
\hline IncRNAs & $\begin{array}{l}\text { Role in } \\
\text { response }\end{array}$ & Targets & Mechanisms & Cancers & Ref \\
\hline AC023II5.3 & $\mathrm{S}$ & miR-26a/GSK3 $\beta$ & ceRNA & Glioblastoma & 76 \\
\hline AK022798 & $\mathrm{R}$ & MRPI, P-gP & $N / A$ & Gastric cancer & 39 \\
\hline AKI 26698 & $\mathrm{R}$ & Wnt/ $\beta$-catenin & $\mathrm{N} / \mathrm{A}$ & NSCLC & 69 \\
\hline ANRIL & $\mathrm{R}$ & MDRI, MRPI & $N / A$ & Gastric cancer & 41 \\
\hline ANRIL & $\mathrm{R}$ & let-7a & $\mathrm{N} / \mathrm{A}$ & $\begin{array}{l}\text { Nasopharyngeal } \\
\text { carcinoma }\end{array}$ & 50 \\
\hline CASC2 & $S$ & miR-2I/PTEN & ceRNA & Cervical cancer & 80 \\
\hline ENST00000457645 & $S$ & Bax, caspase- 3 & $N / A$ & Ovarian cancer & 59 \\
\hline GAS5 & $S$ & miR-2I/PI3K/Akt & ceRNA & Cervical cancer & 83 \\
\hline GAS5 & $S$ & miR-2I/PTEN & ceRNA & NSCLC & 81 \\
\hline HOTAIR & $\mathrm{R}$ & NF- $\kappa B$ & By decreasing $\mid \kappa-B \alpha$ & Ovarian cancer & 48 \\
\hline HOTAIR & $\mathrm{R}$ & $\mathrm{p} 2 \mathrm{I}^{\text {Wafl/Cipl }}$ & $N / A$ & Lung adenocarcinoma & 49 \\
\hline HOTAIR & $\mathrm{R}$ & Beclin-I, MDR, and P-gP & $\mathrm{N} / \mathrm{A}$ & Endometrial cancer & 74 \\
\hline HOTAIR & $\mathrm{R}$ & KIf4 & $\mathrm{N} / \mathrm{A}$ & NSCLC & 78 \\
\hline HOTTIP & $\mathrm{R}$ & Wnt/ $\beta$-catenin & N/A & Osteosarcoma & 71 \\
\hline $\mathrm{HI9}$ & $\mathrm{R}$ & NRF2 & $\mathrm{N} / \mathrm{A}$ & Ovarian cancer & 46 \\
\hline $\mathrm{HIO}$ & $\mathrm{R}$ & FAS, BAK, BAX & $\mathrm{N} / \mathrm{A}$ & Lung adenocarcinoma & 58 \\
\hline LINC00I6I & $S$ & miR-645/IFIT2 & ceRNA & Osteosarcoma & 79 \\
\hline MEG3 & $S$ & $\mathrm{p} 53, \beta$-catenin, survivin, $\mathrm{Bcl}-\mathrm{xl}$ & $N / A$ & Lung cancer & 53,54 \\
\hline MEG3 & $S$ & $\operatorname{miR}-2 \mid 4$ & $N / A$ & Ovarian cancer & 63 \\
\hline MEG3 & $S$ & miR-2I-5p/SOX7 & ceRNA & NSCLC & 82 \\
\hline NEATI & $\mathrm{S}$ & CTRI & ceRNA & Lung cancer & 43 \\
\hline PDAM & $S$ & p53, BCL2LI & $\mathrm{N} / \mathrm{A}$ & Glioma & 51 \\
\hline PVTI & $\mathrm{R}$ & MDRI, MRP, mTOR, HIF-Ia & $\mathrm{N} / \mathrm{A}$ & Gastric cancer & 40 \\
\hline PVTI & $\mathrm{R}$ & TGF- $\beta$ I, p-Smad4, caspase- 3 & $N / A$ & Ovarian cancer & 60 \\
\hline ROR & $\mathrm{R}$ & p53 & $\mathrm{N} / \mathrm{A}$ & $\begin{array}{l}\text { Nasopharyngeal } \\
\text { carcinoma }\end{array}$ & 52 \\
\hline SFTAIP & $\mathrm{S}$ & hnRNP-U-/ADD45A & $N / A$ & LSCC & 64 \\
\hline TRPM2-AS & $\mathrm{R}$ & p53-p66 shc & $\mathrm{N} / \mathrm{A}$ & NSCLC & 56 \\
\hline UCAI & $\mathrm{R}$ & miR-196a & $\begin{array}{l}\text { Affecting transcription } \\
\text { by activating CREB }\end{array}$ & Bladder cancer & 61 \\
\hline UCAI & $\mathrm{R}$ & Caspase-3, CDK2, surviving, p2I & $\mathrm{N} / \mathrm{A}$ & Cervical cancer & 62 \\
\hline UCAI & $\mathrm{R}$ & Wnt $/ \beta$-catenin & $\mathrm{N} / \mathrm{A}$ & Bladder cancer & 70 \\
\hline XIST & $\mathrm{R}$ & miR-I7/ATG7 & ceRNA & NSCLC & 75 \\
\hline
\end{tabular}

Abbreviations: $A B C B I$, ATP-binding cassette subfamily $B$ member I; ABCCI, ATP-binding cassette subfamily C member I; ABCG2, ATP-binding cassette subfamily G member 2; ceRNA, competing endogenous RNA; CREB, cAMP response element-binding protein; CTRI, copper transporter I; IncRNAs, long non-coding RNAs; LSCC, lung squamous cell carcinoma; MDRI, multidrug-resistant protein; MRPI, multidrug-resistant-associated protein I; N/A, not available; NEATI, nuclear-enriched abundant transcript I; NSCLC, non-small-cell lung cancer; P-gp, P-glycoprotein; R, resistance; Ref, reference; S, sensitivity.

cisplatin-resistant and 5-fluorouracil-resistant GC tissues and cells. ${ }^{41}$ Further studies have revealed that ANRIL knockdown might inhibit cell proliferation and invasion, promote anticancer agent-induced apoptosis, and reverse drug resistance in cisplatin- and 5-fluorouracil-resistant GC cell lines by downregulating MDR-related gene expression, including MDR 1 and $M R P 1 .{ }^{41} \mathrm{CTR} 1$, a copper influx transporter, plays a vital role in platinum drug uptake and the development of cisplatin resistance. ${ }^{42}$ In lung cancer cells, lncRNA nuclearenriched abundant transcript 1 (NEAT1) might enhance cisplatin sensitivity by upregulating (-)-epigallocatechin-3gallate (EGCG)-induced CTR1 expression. ${ }^{43}$ Furthermore, NEAT1 might act as a competing endogenous RNA (ceRNA) of hsa-mir-98-5p to regulate CTR1 expression. ${ }^{43}$

\section{Intracellular detoxification}

GSH is a kind of metallothionein, which shows a much higher affinity to cisplatin than DNA. ${ }^{44}$ Increased GSH synthesis was associated with cisplatin resistance, and GSH depletion increased sensitivity to cisplatin. ${ }^{45}$ As such, overexpression of enzymes involved in GSH synthesis and metabolism participates in the process of cisplatin resistance. The lncRNA H19 was overexpressed in ovarian cancer tissues and correlated with cancer recurrence, whereas H19 knockdown in A2780-DR cells increased their sensitivity to cisplatin treatment with a lower GSH level. H19 contributed to cisplatin resistance by regulating NRF2 and its target proteins including NQO1, GSR, G6PD, GCLC, GCLM, and GSTP1, which are involved in the GSH metabolism pathway. ${ }^{46}$ 


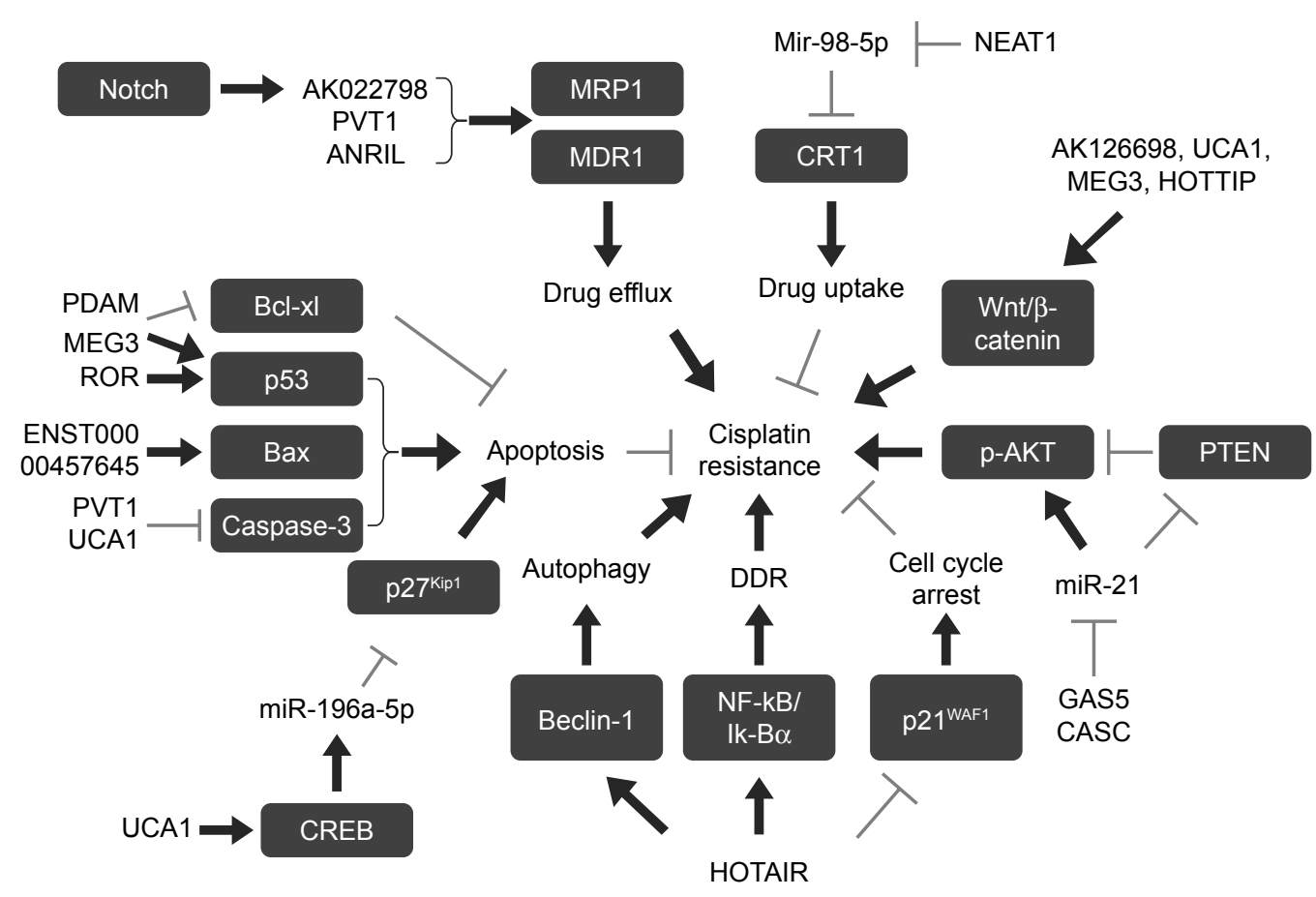

Figure 2 Role of IncRNAs in cisplatin resistance.

Notes: IncRNAs that regulate drug efflux, drug uptake, apoptosis, DDR, cell cycle arrest, and autophagy of cancer cells are implicated in cisplatin resistance. Gray arrows indicate inhibition and black arrows indicate activation.

Abbreviations: DDR, DNA damage response; IncRNAs, long non-coding RNAs; MDRI, multidrug-resistant protein; MRPI, multidrug-resistant-associated protein I.

\section{DNA repair and cell cycle}

Nuclear factor- $\mathrm{\kappa B}(\mathrm{NF}-\mathrm{\kappa B})$ signaling-mediated activation of DNA damage response plays a role in the development of cell resistance to cisplatin. ${ }^{47}$ The lncRNA HOTAIR overexpression induced cisplatin resistance in ovarian cancer cells and resulted in sustained activation of DNA damage response after cisplatin treatment through NF- $\mathrm{\kappa B}$ activation due to I $\kappa-\mathrm{B} \alpha$ (NF- $\kappa \mathrm{B}$ inhibitor) downregulation. Collectively, these data suggests that HOTAIR contributes to chemoresistance through DNA damage-induced NF-KB signaling pathways. ${ }^{48}$ HOTAIR has also been reported to promote cisplatin resistance by regulating $\mathrm{p} 21^{\mathrm{WAF} 1}$ (p21), a cyclin-dependent kinase inhibitor which inhibits cell proliferation by inducing $\mathrm{G} 0 / \mathrm{G} 1$ arrest, in lung adenocarcinoma (LAD) cells. ${ }^{49}$ In nasopharyngeal carcinoma (NPC) cells, knockdown of lncRNA ANRIL inhibited cell proliferation, while it induced cell apoptosis and potentiated cisplatin-induced DNA damage by regulating microRNA let-7a expression. ${ }^{50}$

\section{Apoptosis}

As cisplatin-induced DNA damage causes cell apoptosis, inhibition of apoptosis may also be involved in the acquired cisplatin resistance. $p 53$, a tumor suppressor gene, plays a critical role in the apoptosis pathway. Several studies have shown that IncRNAs were associated with the cisplatin chemoresistance by downregulating p53-induced cell apoptosis. The lncRNA p53-dependent apoptosis modulator (PDAM) silencing induced cisplatin resistance in glioma cells by harboring wild-type p53, while BCL2L1 knockdown in PDAM-suppressed cells abrogated the cisplatin-resistant phenotype ${ }^{51}$ These data indicate that PDAM regulated cisplatin resistance by regulation of $\mathrm{p} 53$-dependent antiapoptotic genes (OTs). ${ }^{51}$ The long non-coding RNA regulator of reprogramming (lncRNA-ROR), which played a crucial role in cell proliferation, migration, and apoptosis of NPC, promoted cisplatin resistance in NPC by improving cell proliferation and reducing cell apoptosis mediated by $\mathrm{p} 53$ signaling pathways. ${ }^{52}$ In A549 cisplatin-resistant cells, lncRNA MEG3 expression was significantly downregulated and overexpression of MEG3 restored cell sensitivity to cisplatin by suppressing cell proliferation and inducing apoptosis and cell cycle arrest. ${ }^{53,54}$ Further studies elucidated that MEG3-mediated chemosensitivity was associated with the WNT/ $\beta$-catenin signaling pathway by regulation of $\mathrm{p} 53$, as well as with the mitochondrial apoptosis pathway. ${ }^{55}$ In addition, Ma et al have revealed that downregulation of lncRNA TRPM2-AS inhibited cisplatin resistance, induced cell apoptosis, and altered cell cycle distribution in NSCLC through activating the $\mathrm{p} 53-\mathrm{p} 66^{\text {shc }}$ pathway. ${ }^{56}$

The Bcl-2 family is a key member in mitochondrial apoptosis pathway, which consists of the antiapoptotic family 
(such as BCL-2 and BCL-XL), the proapoptotic family (BAX and $\mathrm{BAK}$ ), and the proapoptotic $\mathrm{BH} 3$-only protein family (such as BAD, BIK). ${ }^{57}$ The lncRNA H19 contributed to cisplatin resistance in LAD by promoting cell migration via vimentin and reducing apoptosis via FAS, BAK, and BAX. The clinical study has shown that in patients with LAD, a high tumor H19 expression was negatively correlated with cisplatin-based chemotherapy response and a significantly shorter median progression-free survival, which were consistent with the data in in vitro experiment. ${ }^{58}$ The lncRNA ENST00000457645 could remarkably reverse cisplatin resistance by promoting apoptosis of cisplatin-resistant CP70 cells, which was associated with altered levels of apoptosis proteins such as Bax and cleaved caspase- $3 .^{59}$

The lncRNA PVT1 was upregulated in ovarian cancer tissues from cisplatin-resistant patients and in cisplatinresistant cells. PVT1 overexpression promoted cisplatin resistance through regulating the expression of TGF- $\beta 1$, p-Smad4, and caspase-3, molecules related to the apoptotic pathways. ${ }^{60}$ The upregulation of UCA 1 lncRNA contributed to cisplatin resistance by promoting cancer cell proliferation while inhibiting apoptosis in bladder cancer and cervical cancer cells. ${ }^{61,62}$ In human bladder cancer cells, UCA1mediated chemosensitivity was associated with the apoptosis pathway by upregulating miR-196a-5p targeting p27 ${ }^{\text {Kip1 }}{ }^{61}$ In cervical cancer cells, UCA1 suppressed cell apoptosis by downregulating caspase-3 and upregulating CDK2, whereas cell proliferation was enhanced through inducing survivin and decreasing p21 expression. ${ }^{62}$ In ovarian cancer cells, curcumin-induced MEG3 lncRNA expression due to demethylation was directly associated with a decrease in miR-214 and extracellular vesicle-mediated transfer of miR-214, resulting in an elevation of cisplatin-induced cell apoptosis and cell sensitivity to cisplatin-based chemotherapy. ${ }^{63}$ The lncRNA SFTA1P increased cisplatin chemosensitivity by enhancing cisplatin-induced apoptosis by increasing the expression of hnRNP-U and GADD45A in lung squamous cell carcinoma. ${ }^{64}$

It has also been reported that lncRNAs CUDR, HOTAIR, and HULC modulated cisplatin resistance through alteration of cell apoptosis, but their exact molecular mechanisms remain to be elucidated. ${ }^{65-67}$ Wang et $\mathrm{al}^{65}$ have reported that IncRNA CUDR (UCA1a) played a pivotal role in bladder cancer progression, and promoted cell proliferation, migration, and invasion in UM-UC-2 cells. In addition, CUDR overexpression might contribute to cisplatin resistance by antagonizing apoptosis. ${ }^{65}$ HOTAIR also promoted cisplatin resistance in ovarian carcinoma. The knockdown of HOTAIR suppressed cell proliferation and invasion, and notably increased chemosensitivity to cisplatin specifically by promoting cisplatin-induced apoptosis in SKOV-3 cisplatinresistance cells. ${ }^{66}$ Patients with a high expression of HULC lncRNA in GC showed a significantly worse prognosis, and HULC knockdown enhanced the sensitivity of GC cells to cisplatin by enhancing cisplatin-induced apoptosis. ${ }^{67}$

\section{Signaling pathways}

Studies over the years have demonstrated that diverse signaling pathways are involved in the development of drug resistance. ${ }^{68}$ Analysis of mRNA, IncRNA, and miRNA expression profiles by microarray in cisplatin-resistant A549 cells and parental A549 cells revealed that 1,471 mRNAs, 1,380 lncRNAs, and 25 miRNAs were differentially expressed. ${ }^{69}$ Gene coexpression network analysis identified many genes including lncRNA AK126698 that potentially play a significant role in cisplatin resistance. ${ }^{69}$ Pathway analysis showed that the Wnt pathway was targeted by both miRNAs and lncRNAs including lncRNA AK126698. Moreover, in vitro cell culture experiments confirmed that AK126698 lncRNA induced cisplatin resistance in NSCLC through activating Wnt/ $\beta$-catenin pathway. ${ }^{69} \mathrm{UCA} 1 \mathrm{lncRNA}$ expression levels were significantly higher in T24-resistant cells and bladder cancer tissues from patients treated with cisplatin, and overexpression of UCA1 lncRNA promoted cisplatin resistance in bladder cancer cells through upregulating Wnt6 expression, which consequently activated Wnt signaling. ${ }^{70}$ The lncRNA HOTTIP could promote cell proliferation, cell cycle progression, and induce cell resistance to cisplatin by activating the $\mathrm{Wnt} / \beta$-catenin pathway in osteosarcoma and ovarian cancer cells. ${ }^{71}$

\section{Autophagy}

Autophagy plays an important role in the maintenance of cell hemostasis, and LC3, Beclin-1, and Atg family members are important factors in autophagosome formation. ${ }^{72}$ Recently, several studies have reported that autophagy could act as a protective mechanism against cisplatin treatment in cancer cells. ${ }^{29}$ Like 3-MA, an autophagy inhibitor, lncRNA GAS5 was shown to inhibit autophagy and, therefore, enhance cell sensitivity to cisplatin in NSCLC cells. ${ }^{73}$ In human endometrial cancer cells, HOTAIR lncRNA contributed to cisplatin resistance by regulating autophagy mediated through the regulation of Beclin-1 expression. ${ }^{74}$ The lncRNA XIST was upregulated in NSCLC cells and promoted the progression of NSCLC through regulating autophagy. Knockdown of XIST enhanced the chemosensitivity to cisplatin in NSCLC 
cells, which was reversed by the administration of a miR-17 inhibitor and overexpression of ATG7, a key factor in autophagosome formation. These data suggest that lncRNA XIST enhanced the chemoresistance of NSCLC cells to cisplatin by regulating autophagy via the miR-17/ATG7 pathway. ${ }^{75}$ In human glioblastoma cells, the upregulation of lncRNA AC023115.3 promoted chemosensitivity to cisplatin by decreasing autophagy. Further mechanism experiments showed that AC02115.3 acted as a miR-26a sponge and increased its target gene GSK3 $\beta$ expression. ${ }^{76}$

\section{Cancer stem cells (CSCs)}

CSCs are a small population of specialized cells that have the potential to self-renew and differentiate into other tumor cell subtypes and are involved in tumor initiation, progression, distant metastasis, and chemoresistance. Emerging evidence indicates that lncRNAs play an important role in the maintenance of CSCs, increasing tumor cells' resistance to chemotherapy. ${ }^{77}$ HOTAIR lncRNA could promote tumorigenesis and tumor metastasis by affecting the stemness of CSCs. Moreover, Liu et al have found that HOTAIR contributed to cisplatin resistance by regulating the biology of tumor stem cells. ${ }^{78}$ HOTAIR was overexpressed in tumor tissues from NSCLC patients with drug resistance and in cisplatin-resistant A549 cells, and knockdown of HOTAIR expression increased the sensitivity of A549/cisplatin cells to cisplatin. Further mechanistic studies demonstrated that HOTAIR-induced cisplatin resistance might be associated with the promotion of tumor sphere cell growth through upregulating tumor stem cell-related Klf4 expression. ${ }^{78}$

\section{ceRNAs}

In recent years, accumulating evidence indicates that lncRNAs, such as ceRNAs, could regulate target mRNA levels by combining competitively with common miRNAs, which is a potential mechanism in the regulation of cisplatin resistance. The lncRNA NEAT1-enhanced cisplatin sensitivity was mediated through upregulating EGCG-induced CTR1 expression due to its sponging action on mir-98-5p in lung cancer cells. ${ }^{43}$ The lncRNA LINC00161 promoted cisplatininduced apoptosis and decreased cell resistance to cisplatin. Further studies revealed that the effect of LINC00161 was achieved through upregulation of IFIT2 protein expression mediated via competitively sponging miR-645 action on IFIT2 mRNA. ${ }^{79}$ Moreover, other lncRNAs such as CASC, GAS5, and MEG3 have also been reported to function as ceRNAs of miR-21 and miR-21-5p and upregulate PTEN and SOX7 expression, respectively, in NSCLC ${ }^{81,82}$ and cervical cancer cells, ${ }^{80,83}$ resulting in an alteration of cell sensitivity to cisplatin. In glioma cell, IncRNA AC023115.3 acted as a ceRNA for miR-26a and attenuated the inhibitory effect of miR-26a on GSK3 $\beta$, which impaired cisplatin resistance. ${ }^{76}$

\section{Conclusion}

Cisplatin resistance, either intrinsic or acquired, is a significant burden for successful cancer treatment. Here, we have discussed the roles of lncRNAs in cisplatin chemoresistance (Table 1) through mechanisms such as alterations in cellular uptake or efflux of the drug, intracellular detoxification, cell apoptosis, autophagy, DNA repair, CSC, and ceRNA action (Figure 2). Although the study of IncRNAs on chemoresistance is still in its infancy, growing evidence suggests that lncRNAs may serve as biomarkers for cancer diagnosis and prognosis and molecular targets for cancer therapy, including chemoresistance. BC-819 (H19-DTA) is a DNA vector that carries the gene for diphtheria toxin-A under the control of the $\mathrm{H} 19$ promoter sequence, which therefore has the potential to treat various malignancies that overexpress H19 lncRNA. Current clinical trials indicate that $\mathrm{BC}-819$ given locally in combination with systemic chemotherapy may provide an additional therapeutic benefit for the treatment of pancreatic, bladder, ovarian, or peritoneal cancer. ${ }^{84-86}$

Of course, considerable work needs to be done for the lncRNA-based cancer therapy to be applied in clinical practice. First, chemoresistance is a complicated biologic process in which the roles and mechanisms of IncRNAs are still poorly understood. The majority of studies are in in vitro systems. Second, informative functional studies rely on animal experiments. However, establishing lncRNA function model in mice is difficult. Third, the sequence conservation of lncRNAs is much poorer than that of protein-coding genes. Thus, the lncRNAs which have been successfully verified in animal models may be not able to translate into clinical practice. Fourth, as a therapeutic strategy, the technology for either elimination or overexpression of a specific lncRNA at specific target cells in vivo is still in development. Finally, it is currently unclear whether interference of an endogenous IncRNA expression in the body will generate deleterious biologic consequence. Nevertheless, studies over the last decades have provided sufficient data to warrant further investigation of lncRNAs on tumorigenesis, tumor progression, and tumor chemoresistance.

\section{Acknowledgments}

This work was supported in part by the National Natural Science Foundation of China (number 81673516) and a 
Special Talents Fund from the Central South University of China. We are very grateful to Ms Jale Manzo (Department of Medicine, Weill Cornell Medicine, New York, NY, USA) for editing grammar and spelling of the article.

\section{Disclosure}

The authors report no conflicts of interest in this work.

\section{References}

1. Torre LA, Bray F, Siegel RL, Ferlay J, Lortet-Tieulent J, Jemal A. Global cancer statistics, 2012. CA Cancer J Clin. 2015;65(2):87-108.

2. Siegel RL, Miller KD, Jemal A. Cancer statistics, 2018. CA Cancer $J$ Clin. 2018;68(1):7-30.

3. Chen QN, Wei CC, Wang ZX, Sun M. Long non-coding RNAs in anti-cancer drug resistance. Oncotarget. 2017;8(1):1925-1936.

4. Kelland L. The resurgence of platinum-based cancer chemotherapy. Nat Rev Cancer. 2007;7(8):573-584.

5. Huddart RA, Birtle AJ. Recent advances in the treatment of testicular cancer. Expert Rev Anticancer Ther. 2005;5(1):123-138.

6. Artal Cortes A, Calera Urquizu L, Hernando Cubero J. Adjuvant chemotherapy in non-small cell lung cancer: state-of-the-art. Transl Lung Cancer Res. 2015;4(2):191-197.

7. Koberle B, Tomicic MT, Usanova S, Kaina B. Cisplatin resistance: preclinical findings and clinical implications. Biochim Biophys Acta. 2010;1806(2):172-182.

8. Siddik ZH. Cisplatin: mode of cytotoxic action and molecular basis of resistance. Oncogene. 2003;22(47):7265-7279.

9. Rebucci M, Michiels C. Molecular aspects of cancer cell resistance to chemotherapy. Biochem Pharmacol. 2013;85(9):1219-1226.

10. Xiong XD, Ren X, Cai MY, Yang JW, Liu X, Yang JM. Long noncoding RNAs: an emerging powerhouse in the battle between life and death of tumor cells. Drug Resist Updat. 2016;26:28-42.

11. Majidinia M, Yousefi B. Long non-coding RNAs in cancer drug resistance development. DNA Repair (Amst). 2016;45:25-33.

12. Jamieson ER, Lippard SJ. Structure, recognition, and processing of cisplatin-DNA adducts. Chem Rev. 1999;99(9):2467-2498.

13. Rosenberg B, VanCamp L, Trosko JE, Mansour VH. Platinum compounds: a new class of potent antitumour agents. Nature. 1969;222(5191): 385-386.

14. Basu A, Krishnamurthy S. Cellular responses to cisplatin-induced DNA damage. J Nucleic Acids. 2010;2010:201367.

15. Brozovic A. The relationship between platinum drug resistance and epithelial-mesenchymal transition. Arch Toxicol. 2017;91(2):605-619.

16. Ferreira JA, Peixoto A, Neves M, et al. Mechanisms of cisplatin resistance and targeting of cancer stem cells: adding glycosylation to the equation. Drug Resist Updat. 2016;24:34-54.

17. Einhorn LH. Treatment of testicular cancer: a new and improved model. J Clin Oncol. 1990;8(11):1777-1781.

18. Howell SB, Safaei R, Larson CA, Sailor MJ. Copper transporters and the cellular pharmacology of the platinum-containing cancer drugs. Mol Pharmacol. 2010;77(6):887-894.

19. Guminski AD, Balleine RL, Chiew YE, et al. MRP2 (ABCC2) and cisplatin sensitivity in hepatocytes and human ovarian carcinoma. Gynecol Oncol. 2006;100(2):239-246.

20. Galluzzi L, Senovilla L, Vitale I, et al. Molecular mechanisms of cisplatin resistance. Oncogene. 2012;31(15):1869-1883.

21. Arora S, Kothandapani A, Tillison K, Kalman-Maltese V, Patrick SM. Downregulation of XPF-ERCC1 enhances cisplatin efficacy in cancer cells. DNA Repair (Amst). 2010;9(7):745-753.

22. Fink D, Nebel S, Aebi S, et al. The role of DNA mismatch repair in platinum drug resistance. Cancer Res. 1996;56(21):4881-4886.

23. Turner NC, Tutt AN. Platinum chemotherapy for BRCA1-related breast cancer: do we need more evidence? Breast Cancer Res. 2012; 14(6):115.
24. Asada N, Tsuchiya H, Tomita K. De novo deletions of $\mathrm{p} 53$ gene and wild-type p53 correlate with acquired cisplatin-resistance in human osteosarcoma OST cell line. Anticancer Res. 1999;19(6B): $5131-5137$.

25. Leong CO, Vidnovic N, DeYoung MP, Sgroi D, Ellisen LW. The p63/ p73 network mediates chemosensitivity to cisplatin in a biologically defined subset of primary breast cancers. J Clin Invest. 2007;117(5): $1370-1380$.

26. van Oosterwijk JG, Herpers B, Meijer D, et al. Restoration of chemosensitivity for doxorubicin and cisplatin in chondrosarcoma in vitro: BCL-2 family members cause chemoresistance. Ann Oncol. 2012;23(6): $1617-1626$.

27. Henkels KM, Turchi JJ. Cisplatin-induced apoptosis proceeds by caspase-3-dependent and -independent pathways in cisplatin-resistant and -sensitive human ovarian cancer cell lines. Cancer Res. 1999; 59(13):3077-3083.

28. Park MS, De Leon M, Devarajan P. Cisplatin induces apoptosis in LLC-PK1 cells via activation of mitochondrial pathways. J Am Soc Nephrol. 2002;13(4):858-865.

29. Ren JH, He WS, Nong L, et al. Acquired cisplatin resistance in human lung adenocarcinoma cells is associated with enhanced autophagy. Cancer Biother Radiopharm. 2010;25(1):75-80.

30. Mitsuuchi Y, Johnson SW, Selvakumaran M, Williams SJ, Hamilton TC, Testa JR. The phosphatidylinositol 3-kinase/AKT signal transduction pathway plays a critical role in the expression of p21WAF1/CIP1/ SDI1 induced by cisplatin and paclitaxel. Cancer Res. 2000;60(19): 5390-5394.

31. Alexander RP, Fang G, Rozowsky J, Snyder M, Gerstein MB. Annotating non-coding regions of the genome. Nat Rev Genet. 2010;11(8): 559-571.

32. Ma L, Bajic VB, Zhang Z. On the classification of long non-coding RNAs. RNA Biol. 2013;10(6):925-933.

33. Tang Y, Wang J, Lian Y, et al. Linking long non-coding RNAs and SWI/SNF complexes to chromatin remodeling in cancer. Mol Cancer. 2017;16(1):42.

34. Wu R, Su Y, Wu H, Dai Y, Zhao M, Lu Q. Characters, functions and clinical perspectives of long non-coding RNAs. Mol Genet Genomics. 2016;291(3):1013-1033.

35. Gibb EA, Brown CJ, Lam WL. The functional role of long non-coding RNA in human carcinomas. Mol Cancer. 2011;10:38.

36. Zhu QN, Wang G, Guo Y, et al. LncRNA H19 is a major mediator of doxorubicin chemoresistance in breast cancer cells through a cullin4AMDR1 pathway. Oncotarget. 2017;8(54):91990-92003.

37. Du P, Zhao H, Peng R, et al. LncRNA-XIST interacts with miR-29c to modulate the chemoresistance of glioma cell to TMZ through DNA mismatch repair pathway. Biosci Rep. 2017;37(5):BSR20170696.

38. Bian Z, Jin L, Zhang J, et al. LncRNA-UCA1 enhances cell proliferation and 5-fluorouracil resistance in colorectal cancer by inhibiting miR-204-5p. Sci Rep. 2016;6:23892.

39. Hang Q, Sun R, Jiang C, Li Y. Notch 1 promotes cisplatin-resistant gastric cancer formation by upregulating lncRNA AK022798 expression. Anticancer Drugs. 2015;26(6):632-640.

40. Zhang XW, Bu P, Liu L, Zhang XZ, Li J. Overexpression of long noncoding RNA PVT1 in gastric cancer cells promotes the development of multidrug resistance. Biochem Biophys Res Commun. 2015;462(3): 227-232.

41. Lan WG, Xu DH, Xu C, et al. Silencing of long non-coding RNA ANRIL inhibits the development of multidrug resistance in gastric cancer cells. Oncol Rep. 2016;36(1):263-270.

42. Xu X, Ren H, Zhou B, et al. Prediction of copper transport protein 1 (CTR1) genotype on severe cisplatin induced toxicity in non-small cell lung cancer (NSCLC) patients. Lung Cancer. 2012;77(2):438-442.

43. Jiang $\mathrm{P}, \mathrm{Wu} \mathrm{X}$, Wang $\mathrm{X}$, Huang $\mathrm{W}$, Feng Q. NEAT1 upregulates EGCG-induced CTR1 to enhance cisplatin sensitivity in lung cancer cells. Oncotarget. 2016;7(28):43337-43351.

44. Dabrowiak JC, Goodisman J, Souid AK. Kinetic study of the reaction of cisplatin with thiols. Drug Metab Dispos. 2002;30(12):1378-1384. 
45. Jamali B, Nakhjavani M, Hosseinzadeh L, Amidi S, Nikounezhad N, $\mathrm{H}$ Shirazi F. Intracellular GSH alterations and its relationship to level of resistance following exposure to cisplatin in cancer cells. Iran J Pharm Res. 2015;14(2):513-519.

46. Zheng ZG, Xu H, Suo SS, et al. The essential role of H19 contributing to cisplatin resistance by regulating glutathione metabolism in highgrade serous ovarian cancer. Sci Rep. 2016;6:26093.

47. Janssens S, Tinel A, Lippens S, Tschopp J. PIDD mediates NF-kappaB activation in response to DNA damage. Cell. 2005;123(6):1079-1092.

48. Ozes AR, Miller DF, Ozes ON, et al. NF-kappaB-HOTAIR axis links DNA damage response, chemoresistance and cellular senescence in ovarian cancer. Oncogene. 2016;35(41):5350-5361.

49. Liu Z, Sun M, Lu K, et al. The long noncoding RNA HOTAIR contributes to cisplatin resistance of human lung adenocarcinoma cells via downregualtion of p21(WAF1/CIP1) expression. PLoS One. 2013 8(10): e77293.

50. Wang Y, Cheng N, Luo J. Downregulation of lncRNA ANRIL represses tumorigenicity and enhances cisplatin-induced cytotoxicity via regulating microRNA let-7a in nasopharyngeal carcinoma. J Biochem Mol Toxicol. 2017;31(7):e21904.

51. Pang JC, Li KK, Lau KM, et al. KIAA0495/PDAM is frequently downregulated in oligodendroglial tumors and its knockdown by siRNA induces cisplatin resistance in glioma cells. Brain Pathol. 2010;20(6): 1021-1032.

52. Li L, Gu M, You B, et al. Long non-coding RNA ROR promotes proliferation, migration and chemoresistance of nasopharyngeal carcinoma. Cancer Sci. 2016;107(9):1215-1222.

53. Xia Y, He Z, Liu B, Wang P, Chen Y. Downregulation of Meg3 enhances cisplatin resistance of lung cancer cells through activation of the WNT/beta-catenin signaling pathway. Mol Med Rep. 2015;12(3): $4530-4537$.

54. Liu J, Wan L, Lu K, et al. The long noncoding RNA MEG3 contributes to cisplatin resistance of human lung adenocarcinoma. PLoS One. 2015; 10(5):e0114586.

55. Ma W, Li J, Hu J, et al. miR214-regulated p53-NOX4/p66shc pathway plays a crucial role in the protective effect of Ginkgolide B against cisplatin-induced cytotoxicity in HEI-OC1 cells. Chem Biol Interact. 2016;245:72-81.

56. Ma LY, Xie XW, Ma L, et al. Downregulated long non-coding RNA TRPM2-AS inhibits cisplatin resistance of non-small cell lung cancer cells via activation of p53-p66shc pathway. Eur Rev Med Pharmacol Sci. 2017;21(11):2626-2634

57. Youle RJ, Strasser A. The BCL-2 protein family: opposing activities that mediate cell death. Nat Rev Mol Cell Biol. 2008;9(1):47-59.

58. Wang Q, Cheng N, Li X, et al. Correlation of long non-coding RNA H19 expression with cisplatin-resistance and clinical outcome in lung adenocarcinoma. Oncotarget. 2017;8(2):2558-2567.

59. Yan H, Xia JY, Feng FZ. Long non-coding RNA ENST00000457645 reverses cisplatin resistance in CP70 ovarian cancer cells. Genet Mol Res. 2017;16(1):gmr16019411.

60. Liu E, Liu Z, Zhou Y, Mi R, Wang D. Overexpression of long non-coding RNA PVT1 in ovarian cancer cells promotes cisplatin resistance by regulating apoptotic pathways. Int J Clin Exp Med. 2015;8(11):20565-20572.

61. Pan J, Li X, Wu W, et al. Long non-coding RNA UCA1 promotes cisplatin/gemcitabine resistance through CREB modulating miR-196a-5p in bladder cancer cells. Cancer Lett. 2016;382(1):64-76.

62. Wang B, Huang Z, Gao R, et al. Expression of long noncoding RNA urothelial cancer associated 1 promotes cisplatin resistance in cervical cancer. Cancer Biother Radiopharm. 2017;32(3):101-110.

63. Zhang J, Liu J, Xu X, Li L. Curcumin suppresses cisplatin resistance development partly via modulating extracellular vesicle-mediated transfer of MEG3 and miR-214 in ovarian cancer. Cancer Chemother Pharmacol. 2017;79(3):479-487.

64. Li L, Yin JY, He FZ, et al. Long noncoding RNA SFTA1P promoted apoptosis and increased cisplatin chemosensitivity via regulating the hnRNP-U-GADD45A axis in lung squamous cell carcinoma. Oncotarget. 2017;8(57):97476-97489.
65. Wang Y, Chen W, Yang C, et al. Long non-coding RNA UCA1a(CUDR) promotes proliferation and tumorigenesis of bladder cancer. Int J Oncol. 2012;41(1):276-284.

66. Wang Y, Wang H, Song T, et al. HOTAIR is a potential target for the treatment of cisplatinresistant ovarian cancer. Mol Med Rep. 2015; 12(2):2211-2216.

67. Zhang Y, Song X, Wang X, Hu J, Jiang L. Silencing of LncRNA HULC enhances chemotherapy induced apoptosis in human gastric cancer. J Med Biochem. 2016;35(2):137-143.

68. Wickstrom M, Dyberg C, Milosevic J, et al. Wnt/beta-catenin pathway regulates MGMT gene expression in cancer and inhibition of Wnt signalling prevents chemoresistance. Nat Commun. 2015;6:8904.

69. Yang Y, Li H, Hou S, Hu B, Liu J, Wang J. The noncoding RNA expression profile and the effect of lncRNA AK126698 on cisplatin resistance in non-small-cell lung cancer cell. PLoS One. 2013;8(5):e65309.

70. Fan Y, Shen B, Tan M, et al. Long non-coding RNA UCA1 increases chemoresistance of bladder cancer cells by regulating Wnt signaling. FEBS J. 2014;281(7):1750-1758.

71. LiZ,Zhao L, Wang Q. Overexpression of long non-coding RNA HOTTIP increases chemoresistance of osteosarcoma cell by activating the Wnt/ beta-catenin pathway. Am J Transl Res. 2016;8(5):2385-2393.

72. Levine B, Kroemer G. Autophagy in the pathogenesis of disease. Cell. 2008;132(1):27-42.

73. Zhang N, Yang GQ, Shao XM, Wei L. GAS5 modulated autophagy is a mechanism modulating cisplatin sensitivity in NSCLC cells. Eur Rev Med Pharmacol Sci. 2016;20(11):2271-2277.

74. Sun MY, Zhu JY, Zhang CY, et al. Autophagy regulated by lncRNA HOTAIR contributes to the cisplatin-induced resistance in endometrial cancer cells. Biotechnol Lett. 2017;39(10):1477-1484.

75. Sun W, Zu Y, Fu X, Deng Y. Knockdown of lncRNA-XIST enhances the chemosensitivity of NSCLC cells via suppression of autophagy. Oncol Rep. 2017;38(6):3347-3354.

76. Ma B, Yuan Z, Zhang L, et al. Long non-coding RNA AC023115.3 suppresses chemoresistance of glioblastoma by reducing autophagy. Biochim Biophys Acta. 2017;1864(8):1393-1404.

77. Yan $\mathrm{H}, \mathrm{Bu}$ P. Non-coding RNAs in cancer stem cells. Cancer Lett. 2018;421:121-126.

78. Liu MY, Li XQ, Gao TH, et al. Elevated HOTAIR expression associated with cisplatin resistance in non-small cell lung cancer patients. J Thorac Dis. 2016;8(11):3314-3322.

79. Wang Y, Zhang L, Zheng X, et al. Long non-coding RNA LINC00161 sensitises osteosarcoma cells to cisplatin-induced apoptosis by regulating the miR-645-IFIT2 axis. Cancer Lett. 2016;382(2):137-146.

80. Feng Y, Zou W, Hu C, et al. Modulation of CASC2/miR-21/PTEN pathway sensitizes cervical cancer to cisplatin. Arch Biochem Biophys. 2017;623-624:20-30.

81. Cao L, Chen J, Ou B, Liu C, Zou Y, Chen Q. GAS5 knockdown reduces the chemo-sensitivity of non-small cell lung cancer (NSCLC) cell to cisplatin (DDP) through regulating miR-21/PTEN axis. Biomed Pharmacother. 2017;93:570-579.

82. Wang P, Chen D, Ma H, Li Y. LncRNA MEG3 enhances cisplatin sensitivity in non-small cell lung cancer by regulating miR-21-5p/SOX7 axis. Onco Targets Ther. 2017;10:5137-5149.

83. Wen Q, Liu Y, Lyu H, et al. Long noncoding RNA GAS5, which acts as a tumor suppressor via microRNA 21, regulates cisplatin resistance expression in cervical cancer. Int J Gynecol Cancer. 2017;27(6):1096-1108.

84. Hanna N, Ohana P, Konikoff FM, et al. Phase $1 / 2 \mathrm{a}$, dose-escalation, safety, pharmacokinetic and preliminary efficacy study of intratumoral administration of BC-819 in patients with unresectable pancreatic cancer. Cancer Gene Ther. 2012;19(6):374-381.

85. Gofrit ON, Benjamin S, Halachmi S, et al. DNA based therapy with diphtheria toxin-A BC-819: a phase $2 \mathrm{~b}$ marker lesion trial in patients with intermediate risk nonmuscle invasive bladder cancer. J Urol. 2014;191(6):1697-1702.

86. Lavie O, Edelman D, Levy T, et al. A phase 1/2a, dose-escalation, safety, pharmacokinetic, and preliminary efficacy study of intraperitoneal administration of BC-819(H19-DTA) in subjects with recurrent ovarian/ peritoneal cancer. Arch Gynecol Obstet. 2017;295(3):751-761. 


\section{Publish your work in this journal}

OncoTargets and Therapy is an international, peer-reviewed, open access journal focusing on the pathological basis of all cancers, potential targets for therapy and treatment protocols employed to improve the management of cancer patients. The journal also focuses on the impact of management programs and new therapeutic agents and protocols on

patient perspectives such as quality of life, adherence and satisfaction. The manuscript management system is completely online and includes a very quick and fair peer-review system, which is all easy to use. Visit http://www.dovepress.com/testimonials.php to read real quotes from published authors.

Submit your manuscript here: http://www.dovepress.com/oncotargets-and-therapy-journal 